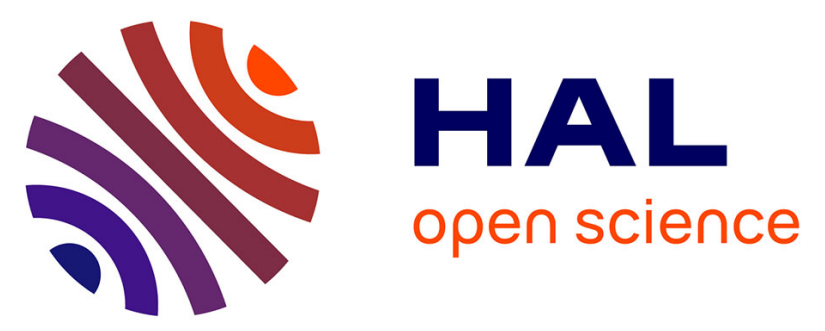

\title{
Genetic analysis of the compatibility between polymerase proteins from human and avian strains of influenza A viruses
}

\author{
Nadia Naffakh, Pascale Massin, Nicolas Escriou, Bernadette \\ Crescenzo-Chaigne, Sylvie van Der Werf
}

\section{To cite this version:}

Nadia Naffakh, Pascale Massin, Nicolas Escriou, Bernadette Crescenzo-Chaigne, Sylvie van Der Werf. Genetic analysis of the compatibility between polymerase proteins from human and avian strains of influenza A viruses. Journal of General Virology, 2000, 81 (5), pp.1283 - 1291. 10.1099/0022-131781-5-1283. pasteur-01611108

\section{HAL Id: pasteur-01611108}

\section{https://hal-pasteur.archives-ouvertes.fr/pasteur-01611108}

Submitted on 5 Oct 2017

HAL is a multi-disciplinary open access archive for the deposit and dissemination of scientific research documents, whether they are published or not. The documents may come from teaching and research institutions in France or abroad, or from public or private research centers.
L'archive ouverte pluridisciplinaire HAL, est destinée au dépôt et à la diffusion de documents scientifiques de niveau recherche, publiés ou non, émanant des établissements d'enseignement et de recherche français ou étrangers, des laboratoires publics ou privés. 


\title{
Genetic analysis of the compatibility between polymerase proteins from human and avian strains of influenza $A$ viruses
}

\author{
Nadia Naffakh, Pascale Massin, Nicolas Escriou, Bernadette Crescenzo-Chaigne \\ and Sylvie van der Werf \\ Unité de Génétique Moléculaire des Virus Respiratoires, URA CNRS 1966, Institut Pasteur, 25 rue du Dr Roux, 75724 Paris Cedex 15 , \\ France
}

In order to determine how efficiently the polymerase proteins derived from human and avian influenza A viruses can interact with each other in the context of a mammalian cell, a genetic system that allows the in vivo reconstitution of active ribonucleoproteins was used. The ability to achieve replication of a viral-like reporter RNA in COS-1 cells was examined with heterospecific mixtures of the core proteins (PB1, PB2, PA and NP) from two strains of human viruses (A/Puerto Rico/8/34 and $A /$ Victoria/3/75), two strains of avian viruses (A/Mallard/NY/6750/78 and A/FPV/Rostock/34), and a strain of avian origin (A/Hong Kong/156/97) that was isolated from the first human case of H5N1 influenza in Hong Kong in 1997. In accordance with published observations on reassortant viruses, PB2 amino acid 627 was identified as a major determinant of the replication efficiency of heterospecific complexes in COS-1 cells. Moreover, the results showed that replication of the viral-like reporter RNA was more efficient when PB2 and NP were both derived from the same avian or human virus or when PB1 was derived from an avian virus, whatever the origin of the other proteins. Furthermore, the PB1 and PB2 proteins from the A/HongKong/156/97 virus exhibited intermediate properties with respect to the corresponding proteins from avian or human influenza viruses, suggesting that some molecular characteristics of PB1 and PB2 proteins might at least partially account for the ability of the A/Hong Kong/156/97 virus to replicate in humans.

\section{Introduction}

Influenza A viruses have been isolated from a wide range of animal species. Aquatic birds, in which all 15 haemagglutinin (HA) subtypes have been found, are believed to be the reservoir of influenza A viruses from which new virus subtypes can episodically be transmitted to new hosts (Webster \& Bean, 1998). Generally, avian influenza viruses (AIVs) replicate poorly in humans (Beare \& Webster, 1991) and in other primates (Murphy et al., 1982). In contrast, reassortants that derive their gene segments from both human and avian viruses are known to be potentially threatening to humans, as such viruses were responsible for the influenza pandemics in 1957 and 1968 (Scholtissek et al., 1978). Segments of avian origin

\footnotetext{
Author for correspondence: Sylvie van der Werf.

Fax +33140613241. e-mail svdwerf@pasteur.fr

The EMBL accession numbers of the sequences reported in this paper are AJ243990-AJ243994.
}

not only encoded novel neuraminidase (NA) and/or HA molecules to which the human population had no immunity, but also the RNA polymerase PBI (Kawaoka et al., 1989). Several observations suggest that avian and human viruses may have reassorted in pigs and that the resulting reassortants may have been transmitted from pigs to humans (Scholtissek et al., 1998). In 1997, a highly pathogenic H5N1 virus that circulated in chickens in Hong Kong infected at least 18 persons and caused six deaths (Centers for Disease Control \& Prevention, 1998). Each case appeared to result from independent transmission of the virus from poultry to humans. There was no convincing evidence for human-to-human transmission and since the mass culling of poultry in Hong Kong, no new human cases of H5N1 virus infection have been recorded. However, related viruses of the H9N2 subtype, with internal genes that showed great similarity with those of the H5N1 Hong Kong viruses, were responsible for two cases of human infection in Hong Kong in 1999, and are still circulating in poultry in Hong Kong (Guan et al., 1999). Therefore, the 
possibility of a productive reassortment between these AIVs and human influenza viruses, which may yield a pandemiogenic reassortant, is a matter of concern. This situation highlights the need for a better understanding of the molecular basis of infectivity of avian/human reassortant influenza A viruses.

For all viral proteins, distinct evolutionary lineages of viral genes are associated with human and avian influenza A viruses (Webster \& Bean, 1998). A number of reassortant viruses which contain human influenza HA and NA genes and various combinations of avian or human influenza internal genes have been generated by coinfection of cultured cells and evaluated for their ability to replicate in vitro and/or in vivo (Tian et al., 1985; Buckler-White et al., 1986; Clements et al., 1986, 1992; Snyder et al., 1987). This has lead to the idea that specific constellations of gene segments encoding the internal proteins could be a determinant for the ability of reassortant viruses to replicate efficiently in mammalian cells and/or in the respiratory epithelium of primates. As far as the core proteins (PB1, PB2, PA and NP) are concerned, studies on reassortant viruses have shown that efficient virus replication is observed for some, but not all, constellations of human and avian virusderived proteins, suggesting that specific interactions between the core proteins are required. On the other hand, coimmunoprecipitation and two-hybrid experiments have indicated that molecular assembly of the polymerase complex relies on PB1-PB2 and PB1-PA interactions, whereas no direct PB2-PA interaction has been detected (Digard et al., 1989; Gonzalez et al., 1996; Toyoda et al., 1996; Zürcher et al., 1996). Biochemical and genetic data have established that the PBI subunit displays RNA-dependent RNA polymerase activity (Braam et al., 1983; Biswas \& Nayak, 1994) and that the PB2 subunit is involved in the cap-snatching mechanism by which viral mRNA synthesis is initiated (Blaas et al., 1982; Braam et al., 1983; Blok et al., 1996; Shi et al., 1996), while the only biochemical activity found to be associated with PA is the induction of proteolysis (Sanz-Ezquerro et al., 1995). Studies on reassortant viruses have clearly shown that the origin of PB2 is a determinant for their efficiency of replication in mammalian cells (Almond, 1977; Clements et al., 1992). A reassortant virus that derived its PB2 gene from the AIV A/Mallard/NY/$6750 / 78$ and its remaining genes from a human virus failed to produce plaques in mammalian cells and its replication was restricted in squirrel monkeys and humans (Clements et al., 1992). Analysis of phenotypic revertants of the reassortant virus indicated that amino acid 627 of PB2 was a major determinant of the phenotype (Subbarao et al., 1993). However, a major limitation from studies on reassortant viruses stems from the fact that all possible constellations among internal genes could not be examined as viruses with certain reassortant genotypes were not isolated, possibly because of a very low replication efficiency.

To address the question as to how efficiently the polymerase proteins derived from a human and an avian virus can interact with each other in the context of a mammalian cell, we made use of the genetic system described by Pleschka et al. (1996) for the in vivo reconstitution of functional ribonucleoproteins (RNPs). This was achieved by transfecting into COS-1 cells a plasmid that directed the expression of a viral-like reporter RNA together with plasmids from which the four core proteins were expressed. The synthetic reporter RNA contained the CAT reporter gene sequence in antisense orientation flanked by the non-coding sequences that serve as promoters for the viral polymerase, which are highly conserved among influenza A viruses (Desselberger et al., 1980). Homospecific as well as heterospecific RNPs were reconstituted by expressing proteins derived (i) from two strains of human viruses, A/Puerto Rico/8/34 (which is anterior to the 1957 reassortment event) and A/Victoria/3/75 (which derives from the 1968 reassortant virus); (ii) from two strains of AIVs, the A/Mallard/NY/6750/78 strain, which was used in the reassortment experiments mentioned above (Clements et al., 1992; Subbarao et al., 1993), and A/FPV/Rostock/34, which is known to be highly pathogenic in chickens; (iii) from $\mathrm{A} /$ Hong Kong/156/97, the H5N1 strain of avian origin that was isolated from the index case in Hong Kong (Claas et al., 1998; Subbarao et al., 1998). This experimental system allowed us to examine how efficiently all possible heterospecific mixtures of the four core proteins permitted transcription/ replication of the viral-like reporter RNA.

\section{Methods}

- Plasmids. Plasmid pHMG contains a mouse hydroxymethylglutaryl coenzyme A reductase (HMG) promoter and a simian virus 40 polyadenylation signal. It was kindly provided by J. Pavlovic (Institut für Medizinische Virologie, Zurich, Switzerland) together with plasmids pHMG-PR8-PB1, pHMG-PR8-PB2, pHMG-PR8-PA and pHMG-PR8$\mathrm{NP}$, which were used to express the PB1, PB2, PA and NP of influenza virus A/Puerto Rico/8/34 (PR8) under the control of the HMG promoter. Plasmids pGEM-VIC-PB1, pGEM-VIC-PB2, pGEM-VIC-PA and pGEM-VIC-NP were kindly provided by A. Portela (Instituto de Salud Carlos III, Madrid, Spain). The cDNAs encoding the PB1, PB2, PA and NP of influenza virus A/Victoria/3/75 (VIC) were transferred from the pGEM-3-derived plasmids to the EcoRV site of plasmid pHMG. Plasmid pPolI-CAT-RT (Pleschka et al., 1996) was kindly provided by P. Palese (Mount Sinaï Medical Center, New York, USA). It contains the CAT gene open reading frame in negative orientation, flanked by the $5^{\prime}$ and $3^{\prime}$ extremities of the NS segment of influenza virus A/WSN/33. Expression of the influenza virus-like RNA is driven by a truncated human RNA Pol I promoter. The correct $3^{\prime}$ end is ensured by the use of the hepatitis delta virus ribozyme sequence.

Molecular cloning and sequencing of cDNAs. Virus isolates A/FPV/Rostock/34 (FPV), A/Mallard/NY/6750/78 (MAL) and A/Hong Kong/156/97 (HK) were grown in 11-day-old embryonated chicken eggs (FPV, MAL) or on MDCK cells (HK). Viral genomic RNA was extracted using $5 \mathrm{M}$ guanidium isothiocyanate and phenol. Fulllength cDNAs were prepared by reverse-transcription of the viral RNA using a primer complementary to the $12 \mathrm{bp}$ conserved at the $3^{\prime}$ end of each viral segment and $20 \mathrm{U}$ avian myeloblastosis virus reverse transcriptase with incubation at $42{ }^{\circ} \mathrm{C}$ for $1 \mathrm{~h}$ followed by incubation at $55{ }^{\circ} \mathrm{C}$ for $15 \mathrm{~min}$. Amplification was performed next using primers 
specific for the coding sequences of PB1, PB2, PA or NP, with additional nucleotides providing an EcoRV site at their $5^{\prime}$ end. The exact sequence of the primers can be obtained from the authors upon request. The PCR conditions were optimized for each sequence to be amplified: either Pwo or Expand High Fidelity polymerase (Boehringer Mannheim) were used, for 30 cycles, each consisting of $1 \mathrm{~min}$ at $94{ }^{\circ} \mathrm{C}, 1 \mathrm{~min}$ at $50-60{ }^{\circ} \mathrm{C}$ (depending on the sequence), 2-9 min (depending on the sequence) at $72{ }^{\circ} \mathrm{C}$. The PCR products were digested with EcoRV and cloned into the EcoRV site of plasmid pGEM5z $(\mathrm{f}+)$. At least three positive clones were sequenced using a dRhod or a Big Dye terminator sequencing kit (Perkin Elmer) and run on a 377 automated sequencer (Perkin Elmer). Clones without PCR-induced mutations were selected, and the EcoRV fragments derived from these were sub-cloned into the EcoRV site of plasmid pHMG.

Construction of mutants. The PCR-based protocol from Tomic et al. (1990) was used to introduce point mutations at residue 627 (Gln $\rightarrow$ Lys) of MAL- and FPV-PB2. Primers were designed to provide two intermediate $\mathrm{PCR}$ products with a region of common sequence containing the mutation at codon 627 (GAA or GAG $\rightarrow$ AAA) and a site for BsaI. The exact sequence of the primers can be obtained from the authors upon request. Plasmids pGEM5z-MAL-PB2 or pGEM5z-FPV-PB2 were used as the starting template. Proof-reading Pwo polymerase (Boehringer Mannheim) was used for PCR amplification, for 20 cycles, each consisting of $1 \mathrm{~min}$ at $94{ }^{\circ} \mathrm{C}, 1 \mathrm{~min}$ at $50{ }^{\circ} \mathrm{C}, 90 \mathrm{~s}$ at $72{ }^{\circ} \mathrm{C}$. The PCR products were digested with $B s a \mathrm{I}$ and ligated to each other. The ligation products were sub-cloned either in pGEM5z-MAL-PB2 using MfeI and EcoRI restriction sites or in pGEM5z-FPV-PB2 using two EcoRI sites. Positive clones were sequenced as previously indicated. Clones bearing the site-directed mutation but no unanticipated nucleotide change were selected, and the EcoRV fragments derived from these were sub-cloned into the EcoRV site of plasmid pHMG.

Transfections and CAT assays. COS- 1 cells were grown in Dulbecco's modified Eagle medium (DMEM) containing 10\% foetal calf serum (FCS). Subconfluent monolayers of COS-1 cells $\left(3 \times 10^{5}\right.$ cells in $35 \mathrm{~mm}$ dishes) were transfected using the Fugene 6-mediated method (Boehringer Mannheim). For the transient replication assay, a mixture of plasmids pHMG-PB1, -PB2, -PA, -NP $(1 ; 1 ; 1 ; 2 \mu \mathrm{g})$ and pPolI-CAT-RT $(1 \mu \mathrm{g})$ was transfected. Cells were incubated at $37^{\circ} \mathrm{C}$ in DMEM containing $10 \%$ FCS until harvest. At 48 h post-transfection, cell extracts were prepared in $500 \mu \mathrm{l}$ of lysis buffer and tested for CAT levels using the CAT ELISA kit (Boehringer Mannheim), which allowed detection of $0.05 \mathrm{ng} / \mathrm{ml}$ CAT. All experiments were done in duplicate.

Western blotting. For Western blot assays, total cell extracts were used. Transfected cells were resuspended directly in $300 \mu$ l of sample loading buffer, the proteins were resolved by electrophoresis in SDS-polyacrylamide gels and transferred onto a nitrocellulose membrane (Biorad). The filters were incubated for $\mathrm{Ih}$ at room temperature or overnight at $4{ }^{\circ} \mathrm{C}$ with primary antibodies diluted in PBS containing $0.1 \%$ BSA (Sigma) and 0.25\% Tween 20. Rabbit antisera directed against the PB1, PB2, and PA proteins of VIC virus were kindly provided by J. Ortin (Universidad Autonoma de Madrid, Spain) and were diluted $1 / 1000$ (PB1, PA) or 1/5000 (PB2). For NP, a mouse antiserum was prepared by repeated intramuscular injection of plasmid pHMG-PR8-NP in mice, and used at a $1 / 500$ dilution. After washing, the filters were incubated for $\mathrm{Ih}$ at room temperature with peroxidase-conjugated secondary antibodies (donkey anti-rabbit-peroxidase or goat antimouse-peroxidase, Amersham) diluted 1/10000 in PBS containing 0.1\% BSA and $0 \cdot 25 \%$ Tween 20 . After washing, the filters were incubated with peroxidase substrate (ECL Plus System, Amersham), scanned using a
STORM820 optical scanner (Molecular Dynamics) and analysed using the Image Quant program (Molecular Dynamics).

\section{Results \\ Functional expression of the core proteins of human and avian influenza viruses}

The cDNAs of the core proteins (PB1, PB2, PA and NP) of the AIV strains FPV, MAL and HK were cloned into the pHMG expression vector as described in Methods, and their sequences were determined. Few or no amino acid differences were found with previously published sequences for MAL proteins (MAL-PB2: Pro $\rightarrow$ Ser 409, Ala $\rightarrow$ Gly 590; MALNP: Pro $\rightarrow$ Ala 336) and HK proteins (HK-PBI: Arg $\rightarrow$ Lys 386; HK-PB2: Met $\rightarrow$ Val 202). In contrast, FPV-PB2, -PA and -NP sequences determined by us showed, respectively, five (out of 759), 15 (out of 716) and four (out of 498) amino acid differences as compared to the previously published sequences; for this reason they were submitted to the EMBL database (accession number for influenza A/FPV/Rostock/34 PB2, AJ243991; influenza A/FPV/Rostock/34 PA, AJ243992; influenza A/FPV/Rostock/34 NP, AJ243993). Sequences of FPV-PBI and MAL-PA had not been published before (EMBL accession number for influenza A/FPV/Rostock/34 PB1, AJ243990; influenza A/Mallard/NY/6750/78 PA, AJ243994). They respectively showed great similarity with available PBI sequences (at least $87 \cdot 6 \%$ at the nucleotide level and $97.3 \%$ at the amino acid level) and PA sequences (at least $89.5 \%$ at the nucleotide level and $98.2 \%$ at the amino acid level) from other avian virus strains. Alignment of all available sequences for PB1, PB2, PA or NP revealed that at specific positions some amino acids appeared to be characteristic of the human or avian origin of the sequences (Fig. 1). Interestingly, in the HK-derived sequence, human-type residues were observed at some of these specific positions (PB2 Ser 199, PB1 Arg 54, PA Leu 400 and Asn 409, NP Met 136). However, only a limited number of avian PB1, PB2 and PA sequences were available for alignment.

In COS-1 cells transfected with the pHMG-PR8-PBI, -PB2, -PA and -NP plasmids together with plasmid pPolICAT-RT, high levels of CAT expression were measured $(2441 \pm 2 \mathrm{ng} / \mathrm{ml})$, as expected from previously published work (Pleschka et al., 1996). Comparable levels of CAT expression were achieved when VIC or FPV replication complexes were reconstituted ( $2439 \pm 101$ and $2203 \pm 89 \mathrm{ng} / \mathrm{ml}$, respectively), whereas reduced levels were repeatedly observed for MAL and HK complexes $(834 \pm 152$ and $1084 \pm 161 \mathrm{ng} / \mathrm{ml}$, respectively). When cell extracts were analysed by Western blot, using PB1-, PB2-, PA- or NP-specific antisera, all the recombinant polypeptides could be detected with their expected size (not shown). Furthermore, comparable levels of expression were observed irrespective of the virus from which the proteins were derived. The observed variations in CAT production might therefore reflect a lower specific activity of the MAL and HK complexes. 


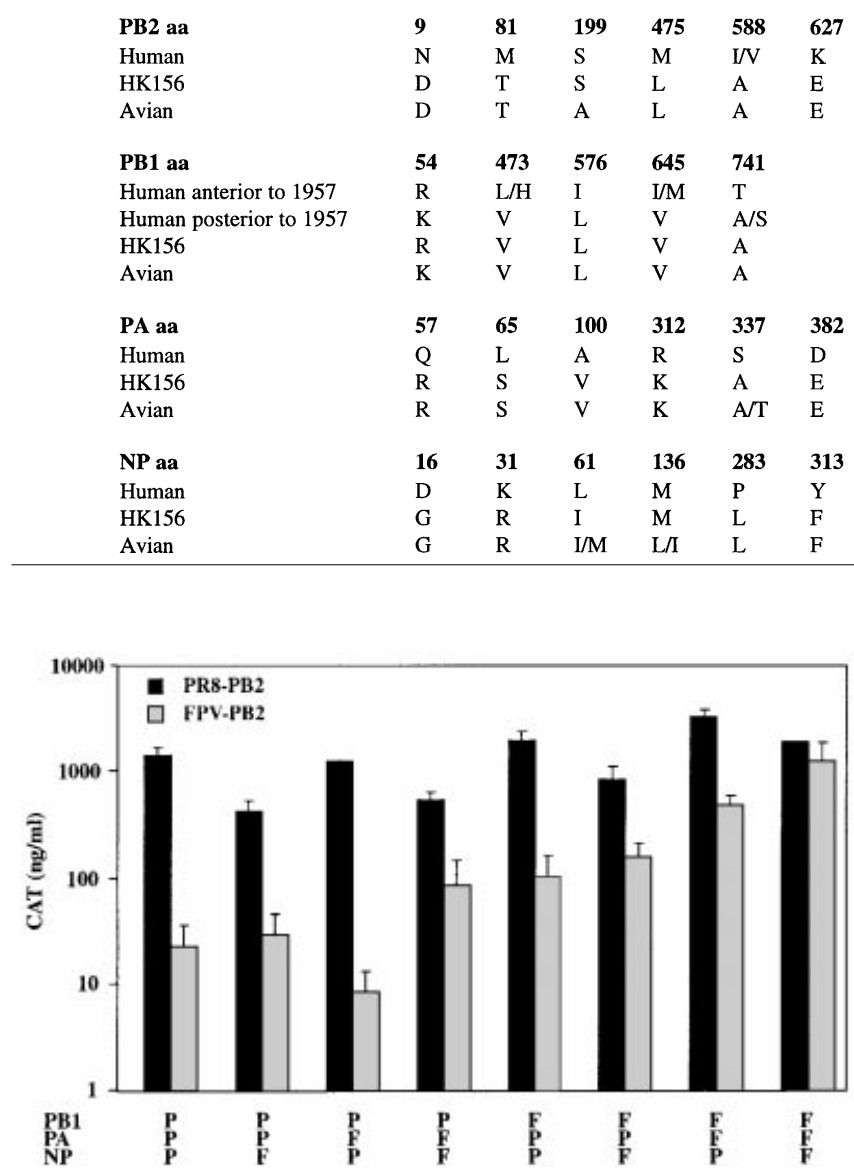

Fig. 2. Effect of the human or avian origin of PB2 on the expression of the viral-like reporter RNA. COS-1 cells were transfected in duplicate with the 16 possible combinations between plasmids encoding the four PR8- and FPV-derived core proteins, together with the pPoll-CAT-RT plasmid. At $48 \mathrm{~h}$ post-transfection, cell extracts were prepared and tested for the levels of CAT as described in Methods. For a given combination of FPV (F)- and PR8 (P)-derived PB1, PA and NP, the CAT levels measured when PB2 was derived from FPV (grey bars) were compared to the CAT levels measured when PB2 was derived from PR8 (black bars). The results are expressed as the average $\pm \mathrm{SD}$ of two independent experiments.

\section{Functional analysis of heterospecific replication complexes}

In order to determine how efficiently heterospecific mixtures of PB1, PB2, PA and NP allowed transcription/ replication of the viral-like reporter RNA, heterospecific RNPs were reconstituted with proteins derived from either human (PR8, VIC), avian (FPV, MAL) or HK viruses. For a given pair of viruses, all 16 possible combinations between the two sets of pHMG-PB1, -PB2, -PA and -NP plasmids were transfected together with plasmid pPolI-CAT-RT into COS-I cells. The efficiency with which the various reconstituted heterospecific complexes permitted transcription/replication of the viral-like reporter RNA was determined by measuring the CAT levels in
Fig. 1. Amino acids characteristic of the human or avian origin of influenza $A$ virus core proteins. Number of strains used in this analysis: PB1, four human anterior to 1957, 15 human posterior to 1957, 12 avian; PB2, 20 human, 14 avian; PA, 15 human, 13 avian; NP, 45 human, 53 avian. HK156, A/Hong Kong/156/97 strain. Avian sequences do not include the A/Chicken/Hong Kong/-220/97, -G23/97, -G9/97, A/Chicken/Korea/25232/96, -38349/96, A/Duck/Hong Kong/-Y280/97, -Y439/97, A/Pigeon/Hong Kong/Y233/97 and A/Quail/Hong Kong/G1/97 sequences, which were found to be very close to HK156 sequences (Suarez et al., 1998; Guan et al., 1999) and could therefore be considered as possibly intermediate between pure avian and pure human viruses. extracts prepared from transfected cells. In order to analyse separately the effects of the origin of PB2, PB1, PA or NP, we studied successively the variations of CAT levels that resulted from the permutation of one of the four proteins from one strain to another.

The human or avian origin of PB2 appeared to be a determinant for the efficiency with which heterospecific complexes allowed transcription/replication of the viral-like reporter RNA. Indeed, whatever the combination of PBI, PA and NP between PR8 and FPV viruses, high levels of CAT expression $(>600 \mathrm{ng} / \mathrm{ml})$ were measured in cell extracts when PB2 was derived from the human virus (PR8). Furthermore, except when PB1, PA and NP were of avian origin, CAT expression was systematically higher (5-100-fold) when PB2 was derived from the human (PR8) rather than from the avian (FPV) virus (Fig. 2). Very similar results were obtained when PR8/MAL or VIC/FPV protein combinations were tested (not shown). In contrast, PB2 permutations had little effect among the MAL/FPV combinations (not shown). As amino acid 627 of PB2 (Glu in PB2 proteins of avian origin, Lys in PB2 proteins of human origin) has been identified as an important determinant of the host range of human/avian reassortant viruses (Subbarao et al., 1993), we generated cDNAs encoding PB2 from MAL or FPV viruses with a Glu $\rightarrow$ Lys substitution at residue 627 (E627K), by site-directed mutagenesis. Western blot analysis showed that the wild-type and mutant proteins were expressed at similar levels (Table 1 ). When the mutant MAL- or FPV-PB2 proteins were used in the replication assay, the levels of CAT expression were drastically increased as compared to the levels observed with the corresponding avian PB2 proteins (Table 1). In the case of the combination where avian PB1, human PA and avian NP were used, the levels of CAT expression reached with the E627K mutant PB2 proteins were similar to those observed with PR8PB2 (Table 1). This confirmed that PB2 amino acid 627 is an important determinant of the ability of avian/human hetero- 
Table 1. Effect of the human or avian origin of PB2 on expression of the viral-like reporter RNA

\begin{tabular}{|c|c|c|c|c|c|c|}
\hline PB1 & PA & NP & PB2 & Western blot* & CAT (ng/ml) + & CAT (\%)‡ \\
\hline- & - & - & - & & $<0.05$ & \\
\hline PR8 & PR8 & PR8 & PR8 & 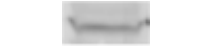 & $2207 \pm 175$ & 100 \\
\hline PR8 & PR8 & PR8 & MAL & 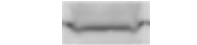 & $16 \pm 3$ & $0.7 \pm 0.05$ \\
\hline PR8 & PR8 & PR8 & MAL-E627K & 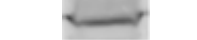 & $618 \pm 42$ & $28 \pm 2$ \\
\hline PR8 & PR8 & PR8 & FPV & 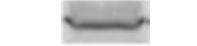 & $21 \pm 0 \cdot 5$ & $0.9 \pm 0.05$ \\
\hline PR8 & PR8 & PR8 & FPV-E627K & 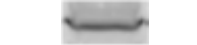 & $959 \pm 140$ & $43 \pm 18$ \\
\hline PR8 & PR8 & PR8 & HK & 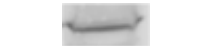 & $6 \pm 1$ & $0.3 \pm 0.02$ \\
\hline MAL & PR8 & MAL & PR8 & & $1518 \pm 76$ & 100 \\
\hline MAL & PR8 & MAL & MAL & & $235 \pm 22$ & $15 \pm 1$ \\
\hline MAL & PR8 & MAL & MAL-E627K & & $1634 \pm 28$ & $108 \pm 2$ \\
\hline FPV & PR8 & FPV & PR8 & & $886 \pm 69$ & 100 \\
\hline FPV & PR8 & FPV & FPV & & $303 \pm 2$ & $34 \pm 0.05$ \\
\hline FPV & PR8 & FPV & FPV-E627K & & $1219 \pm 162$ & $138 \pm 18$ \\
\hline HK & PR8 & HK & PR8 & & $845 \pm 51$ & 100 \\
\hline HK & PR8 & HK & HK & & $434 \pm 43$ & $50 \pm 5$ \\
\hline
\end{tabular}

* For Western blot analysis, COS-I cells were transfected in duplicate with the pHMG control plasmid (first line) or the pHMG plasmids encoding either PR8-, MAL-, MAL-E627K- FPV-, FPV-E627K-, or HK-PB2. At $48 \mathrm{~h}$ post-transfection, cell extracts were prepared and analysed by Western blot using a polyclonal serum specific for PB2 as described in Methods. The image obtained by scanning the blot with a STORM820 optical scanner is shown.

+ CAT levels are expressed as the mean $\pm S D$ values from one representative experiment in duplicate, out of two.

\# For a given combination of PB1, PA and NP, CAT levels measured when PB2 was of avian origin are expressed as the percentage of the CAT level measured when PB2 was derived from PR8 virus.

specific RNPs to undergo transcription/replication in mammalian cells. When PR8 and HK proteins were mixed, substitutions of PR8-PB2 by HK-PB2 generally had the same effects as substitution of PR8-PB2 by MAL- or FPV-PB2 (not shown). However, in a number of combinations, such as the avian- or HK-PBI, PR8-PA, avian- or HK-NP combination shown in Table 1 , we repeatedly observed that CAT expression was reduced to a lesser extent when PR8-PB2 was replaced by HK-PB2 rather than by MAL- or FPV-PB2 $(50 \%$ reduction compared to 85 and $66 \%$ reduction respectively, $P<0 \cdot 1$ ).

The human or avian origin of PBI did not appear to be as strong a determinant as the origin of PB2 for the efficiency with which heterospecific complexes permitted transcription/ replication of the viral-like reporter RNA. The differences between the CAT levels measured in the presence of PBI derived from either the human PR8 or the avian FPV virus were generally less than 5-fold (Fig. 3). However, they were systematically in favour of FPV-PB1. The CAT levels observed with PR8-PB1 were especially low as compared to FPV-PBI in the combinations in which PB2 was derived from FPV, which could possibly result from inefficient PR8-PB1/FPV-PB2 interactions. However, the CAT levels observed with PR8-PB1 were also lower than the levels obtained with FPV-PBI in combinations in which most of the proteins were derived from PR8 (Fig. 3). Similarly, for heterospecific replication complexes derived from the human PR8 and avian MAL viruses, the CAT levels were consistently higher with the avian MAL-PBI relative to the human PR8-PBI (not shown). Interestingly, permutations between VIC- and FPV-PBI, as well as between MAL- and FPV-PB1, in heterospecific VIC/FPV or MAL/FPV replication complexes, respectively, had no significant effect on CAT levels (not shown). This observation is consistent with the fact that, unlike PR8, the human VIC strain is derived from the 1968 reassortant virus whose PBI segment was acquired from an avian virus. When proteins from PR8 and HK were mixed, for several combinations of PB2, PA and NP, the augmentation of CAT levels observed when HK-PBI was used instead of PR8-PB1 was not as marked as the increase observed with FPV-PBI relative to PR8-PBI (Fig. 3 b).

The origin of NP had only a slight effect on the functional efficiency of PR8/FPV heterospecific complexes for the 

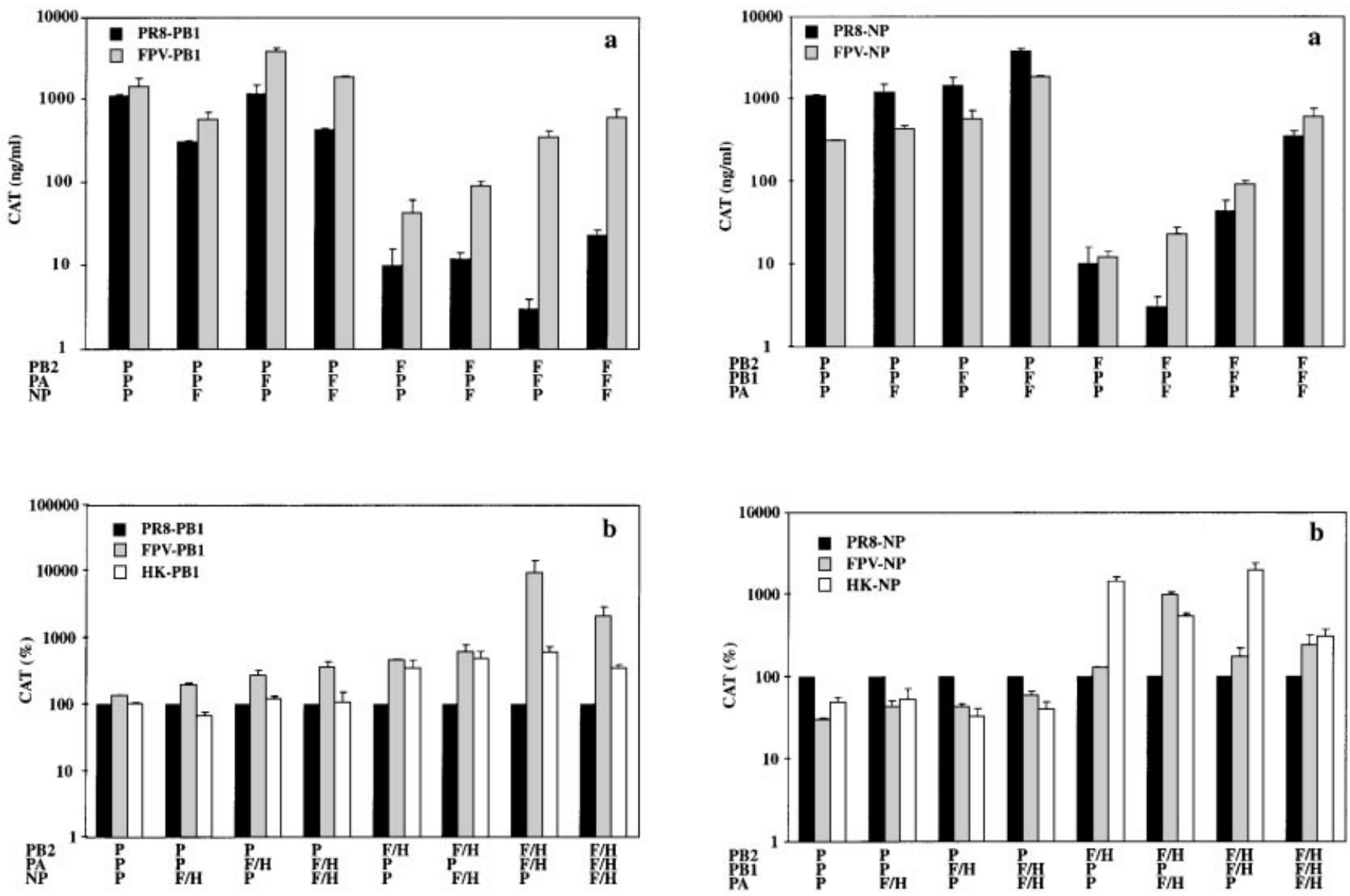

Fig. 3. Effect of the human or avian origin of PB1 on the expression of the viral-like reporter RNA. COS-1 cells were transfected in duplicate with the 16 possible combinations between plasmids encoding the four PR8- and FPV-derived core proteins, or between plasmids encoding the four PR8and HK-derived core proteins, together with the pPoll-CAT-RT plasmid. At $48 \mathrm{~h}$ post-transfection, cell extracts were prepared and tested for the levels of CAT as described in Methods. (a) For a given combination of FPV (F)- and PR8 (P)-derived PB2, PA and NP, the CAT levels measured when PB1 was derived from FPV (grey bars) were compared to the CAT levels measured when PB1 was derived from PR8 (black bars). The results are expressed as the average \pm SD of one representative out of two independent experiments. (b) For a given combination of FPV (F)- and PR8 (P)-derived PB2, PA and NP, the CAT levels measured when PB1 was derived from FPV (grey bars) were compared to the CAT levels measured when PB1 was derived from PR8 (black bars $=100 \%$ ). For a given combination of HK $(\mathrm{H})$ - and PR8 (P)-derived PB2, PA and NP, the CAT levels measured when PB1 was derived from HK (white bars) were compared to the CAT levels measured when PB1 was derived from PR8 (black bars $=100 \%$ ). The results are expressed as percentage values, and as the average $\pm S D$ of two independent experiments.

transcription/replication of the viral-like reporter RNA. However, whatever the origin of PBI and PA, the CAT levels appeared to be higher when NP and PB2 were derived from the same virus (Fig. 4). Homospecificity of NP and PB2 also appeared to result in enhanced CAT levels when proteins from PR8 and MAL (not shown) or PR8 and HK (Fig. 4b) were mixed. In contrast, NP and PB2 homospecificity had no effect on CAT expression when combinations between FPV and MAL proteins were tested (not shown).

Fig. 4. Effect of the human or avian origin of NP on the expression of the viral-like reporter RNA. COS-1 cells were transfected in duplicate with the 16 possible combinations between plasmids encoding the four PR8- and FPV-derived core proteins, or between plasmids encoding the four PR8and HK-derived core proteins, together with the pPoll-CAT-RT plasmid. At $48 \mathrm{~h}$ post-transfection, cell extracts were prepared and tested for the levels of CAT as described in Methods. (a) For a given combination of FPV (F) - and PR8 (P)-derived PB1, PB2 and PA, the CAT levels measured when NP was derived from FPV (grey bars) were compared to the CAT levels measured when NP was derived from PR8 (black bars). The results are expressed as the average $\pm S D$ of one representative out of two independent experiments. (b) For a given combination of FPV (F)- and PR8 (P)-derived PB1, PB2 and PA, the CAT levels measured when NP was derived from FPV (grey bars) were compared to the CAT levels measured when NP was derived from PR8 (black bars $=100 \%)$. For a given combination of HK $(\mathrm{H})$ - and PR8 $(\mathrm{P})$-derived PB1, PB2 and PA, the CAT levels measured when NP was derived from HK (white bars) were compared to the CAT levels measured when NP was derived from PR8 (black bars $=100 \%$ ). The results are expressed as percentage values, and as the average $\pm \mathrm{SD}$ of two independent experiments.

The effect of the origin of PA on the ability of heterospecific complexes to allow transcription/replication of the viral-like reporter RNA could not be analysed because we observed that the level of proteolytic activity associated with PA (SanzEzquerro et al., 1995) varied from one strain to another (not shown). Since this could affect the level of accumulation of coexpressed influenza virus core proteins and reporter CAT protein, it precluded the analysis of PA permutations in the replication assay. However, it was not likely to interfere with 
the results concerning the role of the other core proteins, as the origin of PA was kept unchanged when permutations of PBI, PB2 or NP were analysed.

\section{Discussion}

It was previously demonstrated that functional influenza Alike RNPs can be reconstituted in vivo by expressing the four core proteins (PB1, PB2, PA and NP) from cloned cDNAs together with a plasmid-derived viral-like RNA (Pleschka et al., 1996). In this report we establish that this exclusively plasmiddriven system can be used for the genetic analysis of the compatibility of core proteins from different strains of influenza A viruses. Homologous complexes, derived either from human viruses (PR8 and VIC), AIVs (MAL and FPV) or a strain of avian origin that was responsible for a fatal respiratory illness in a young boy in Hong Kong in 1997 (HK), allowed efficient transcription/replication of the viral-like reporter RNA in COS- 1 cells. This observation is consistent with the fact that human and avian viruses have been shown to replicate with similar efficiencies in mammalian cells (Clements et al., 1986, 1992; Snyder et al., 1987). It confirms that both avian and human polymerase complexes can assemble correctly (and possibly, interact efficiently with cellular factors) in mammalian cells. We examined how efficiently heterologous mixtures of PB1, PB2, PA or NP allowed transcription/replication of the viral-like reporter RNA in COS-I cells. Important variations in the efficiency of transcription/replication were observed among the combinations of proteins derived from an avian and a human strain, which could possibly reflect some level of incompatibility between these proteins.

The origin of $\mathrm{PB} 2$, and especially the nature of $\mathrm{PB} 2$ amino acid 627, appeared to be an important determinant of the functional efficiency of heterospecific complexes in COS-1 cells. Our observations being in accordance with previous observations on human/avian reassortant viruses (Clements et al., 1992; Subbarao et al., 1993), we believe that they constitute a validation of our experimental approach for the identification of other molecular determinants of the compatibility between core proteins from human and avian strains of influenza A viruses. Moreover, they demonstrate that in order to manifest, the PB2-mediated restriction of transcription/replication in mammalian cells does not need the expression of viral genes other than PB1, PA and NP. Detailed analysis of the RNA species will be needed to determine precisely which transcription and/or replication process is affected by the origin of PB2. The poor efficiency of heterospecific complexes comprising a PB2 subunit of avian origin could result from an incompatibility with other subunits of human origin and/or an altered interaction with a host factor. If protein incompatibility only was involved, poor efficiency would probably characterize both an avian/human complex and its human/avian counterpart, which does not correspond to our observations. Indeed our analysis of all possible constellations among the polymerase genes (which was not provided by studies on reassortant viruses) shows that in the context of a mammalian cell, PB2 of human origin cooperates efficiently with any combination of PB1, PA or NP proteins. If interaction of PB2 with a host factor was the only mechanism involved, avian homospecific complexes and any heterospecific complex comprising a PB2 protein of avian origin would function with an equally poor efficiency, which does not correspond to our observations either. Indeed avian-derived homospecific complexes achieved similar levels of transcription/replication of the viral-like reporter RNA as compared to human-derived complexes. On the whole, our observations suggest that the mechanism by which differences in the PB2 protein, and especially the nature of amino acid 627, alter the efficiency of transcription/replication is complex, relying on interactions of PB2 with both viral and cellular proteins. Interaction of PB2 residue 627 with a host factor is likely to be the major determinant, as the efficiencies of the MAL and FPV complexes were increased when MAL-E627K-PB2 and FPV-E627K-PB2 were combined with MAL-PB1, -PA, -NP and FPV-PB1, -PA, -NP, respectively (results not shown). Further experiments involving both mammalian and avian cell lines will probably help clarify this point.

We observed that homospecificity between PB2 and NP appeared to enhance the functional efficiency of chimeric complexes. The NP, in addition to its structural role for the formation of the RNPs, is required for efficient elongation of RNA chains and is thought to be involved in the switch from transcription to replication (Beaton \& Krug, 1986; Honda et al., 1988). In accordance with previously published results based on genetic (Mandler et al., 1991) or biochemical (Biswas et al., 1998) studies, our observations suggest that PB2-NP interactions could be involved in the transcription/replication process, and could therefore impose some constraints on reassortment events that involve the corresponding segments. Replication assays using human/avian chimeric proteins may help identify the domains of interaction between PB2 and NP. In this study, the human or avian origin of NP per se was not found to be a determinant for the efficiency of transcription/ replication of the viral-like reporter RNA in COS-I cells. In the context of a reassortant virus which derived all remaining genes from a human virus, the NP gene from the MAL avian virus was shown to specify restriction of virus replication in squirrel monkeys (Tian et al., 1985; Clements et al., 1992), but not in humans or in MDCK cells (Clements et al., 1992). Our observations suggest that the attenuation which has been observed in squirrel monkeys does not result from an impairment of the transcription/replication process.

In the context of a mammalian cell, PB1 of avian origin appeared to cooperate with PB2, PA and NP of human origin more efficiently than $\mathrm{PBI}$ of human origin appeared to cooperate with PB2, PA and NP of avian origin. In particular, in our experiments the $\mathrm{PB} 2$ human-PB1 avian-PA human constellation (HAH), associated either with a human or avian 
$\mathrm{NP}$, permitted efficient replication of the viral-like RNA reporter. This suggests that the phenotype of influenza A/Pintail/119/79 $\times$ A/Washington/897/80 reassortant viruses, which possessed the $\mathrm{HAH}$ constellation and were restricted in MDCK cells and in monkeys (Snyder et al., 1987), was not determined by the $\mathrm{HAH}$ constellation only, but probably by the origin of the NS and M segments also. Our results may also be related to the fact that segments found to be of avian origin in the 1957 and 1968 pandemic viruses encoded not only the surface glycoproteins (NA and/or HA) but also PBI (Kawaoka et al., 1989). Taken together, these observations suggest that PBI proteins of avian origin might have a higher specific activity than PBI proteins of human origin, and that the acquisition of the PBI segment from an avian virus may have conferred a selective advantage to the 1957 and 1968 reassortant viruses. In an alignment of PB1 sequences of human or avian origin (given the fact that only four sequences, including PR8-PB1, are available for human viruses isolated before 1957), five amino acids appeared as characteristic of the origin of the sequences (Fig. 1). Remarkably, in all PBI sequences derived from human viruses isolated later than 1957, including six virus strains isolated in Japan in 1993-1996 (Lindstrom et al., 1998), avian-type residues were found at all but one of these specific positions (Fig. 1). Conservation over a period of 28 years (running from the 1968 reassortment event up to 1996) may reflect some selective advantage conferred by these avian-type residues. In addition to the four conserved motifs found among all viral RNA-dependent polymerases (Poch et al., 1989), putative nucleotide-binding domains (Asano \& Ishihama, 1997) and RNA-binding sites (Li et al., 1998; Gonzalez \& Ortin, 1999a, b) have recently been identified on PBI. A number of amino acids that appear to be characteristic of the human or avian origin of the sequences are located within these domains and may therefore affect the specific activity of the PBI proteins.

Interestingly, $\mathrm{PB} 2$ and $\mathrm{PB} 1$ proteins from the HK virus were found to behave in an intermediate fashion with respect to the corresponding proteins from human and avian viruses. Indeed, in the replication assay, both the disadvantage of HK-PB2 over PR8-PB2 and the advantage of HK-PB1 over PR8-PB1 were reduced in comparison to FPV or MAL proteins in a number of combinations. As far as PB2 is concerned, the HK strain used here shows a Glu at residue 627 (typical of avian viruses). Our data suggest that some molecular characteristics other than the nature of residue 627 could render HK-PB2 closer to human virus-derived PB2 than FPV or MAL-PB2. A potential candidate is residue 199 that is of the human type (Ser) in HKPB2 (Fig. 1), while it is of the avian type (Ala) in the sequence of PB2 from an H5N1 virus (A/Chicken/Hong Kong/220/97) circulating in chickens in Hong Kong during the same period (Suarez et al., 1998). Mutagenesis experiments will be needed to confirm this hypothesis. The fact that not only one but all three PB1, PB2 and PA proteins from HK show some characteristics of human viruses at the level of their amino acid sequence (Fig. 1) is probably related to the fact that proteins associated physically and functionally undergo co-evolution. Our observations suggest that PBI and PB2 proteins from HK may also have acquired some functional characteristics from human viruses, which could partially account for the ability of this virus strain to replicate in humans.

The authors are very grateful to J. Pavlovic for providing the pHMG recombinant plasmids, to A. Portela for providing the $\mathrm{pGEM}$ recombinant plasmids, to P. Palese for providing plasmid pPolI-CAT-RT, and to J. Ortin for providing polyclonal sera specific for PB1, PB2 and PA proteins. They are grateful to A. Hay for providing the A/FPV/Rostock/34 and A/Hong Kong/156/97 viruses, and R. Webster for providing the A/Mallard/NY/6750/78 virus. The technical assistance of Ida Rijks and Claudine Rousseaux for the production of influenza viruses is gratefully acknowledged. We thank Cécile Quemin for contribution to the initial cloning of the A/Hong Kong/156/97 cDNAs, and Marco Vignuzzi for critical reading of the manuscript.

This work was supported in part by the Ministere de l'Education Nationale, de la Recherche et de la Technologie (EA 302).

\section{References}

Almond, J. W. (1977). A single gene determines the host range of influenza virus. Nature 270, 617-618.

Asano, Y. \& Ishihama, A. (1997). Identification of two nucleotidebinding domains on the PBI subunit of influenza virus RNA polymerase. Journal of Biochemistry 122, 627-634.

Beare, A. S. \& Webster, R. G. (1991). Replication of avian influenza viruses in humans. Archives of Virology 119, 37-42.

Beaton, A. R. \& Krug, R. M. (1986). Transcription antitermination during influenza viral template RNA synthesis requires the nucleocapsid protein and the absence of a $5^{\prime}$ capped end. Proceedings of the National Academy of Sciences, USA 83, 6282-6286.

Biswas, S. K. \& Nayak, D. P. (1994). Mutational analysis of the conserved motifs of influenza A virus polymerase basic protein 1. Journal of Virology 68, 1819-1826.

Biswas, S. K., Boutz, P. L. \& Nayak, D. P. (1998). Influenza virus nucleoprotein interacts with influenza virus polymerase proteins. Journal of Virology 72, 5493-5501.

Blaas, D., Patzelt, E. \& Kuechler, E. (1982). Cap-recognizing protein of influenza virus. Virology 116, 339-348.

Blok, V., Cianci, C., Tibbles, K. W., Inglis, S. C., Krystal, M. \& Digard, P. (1996). Inhibition of the influenza virus RNA-dependent RNA polymerase by antisera directed against the carboxy-terminal region of the PB2 subunit. Journal of General Virology 77, 1025-1033.

Braam, J., Ulmanen, I. \& Krug, R. M. (1983). Molecular model of a eucaryotic transcription complex: functions and movements of influenza P proteins during capped RNA-primed transcription. Cell 34, 609-618.

Buckler-White, A. J., Naeve, C. W. \& Murphy, B. R. (1986). Characterization of a gene coding for $\mathrm{M}$ proteins which is involved in host range restriction of an avian influenza A virus in monkeys. Journal of Virology 57, 697-700.

Centers for Disease Control \& Prevention (1998). Update: isolation of avian influenza A (H5N1) viruses from humans-Hong Kong, 1997-1998. Morbidity \& Mortality Weekly Report 46, 1245-1247.

Claas, E. C., Osterhaus, A. D., van Beek, R., De Jong, J. C., Rimmelzwaan, G. F., Senne, D. A., Krauss, S., Shortridge, K. F. \& Webster, R. G. (1998). Human influenza A H5N1 virus related to a 
highly pathogenic avian influenza virus [published erratum appears in Lancet (1998) 351, 1292]. Lancet 351, 472-477.

Clements, M. L., Snyder, M. H., Buckler-White, A. J., Tierney, E. L., London, W. T. \& Murphy, B. R. (1986). Evaluation of avian-human reassortant influenza $A /$ Washington/897/80 $\times \mathrm{A} /$ Pintail/119/79 virus in monkeys and adult volunteers. Journal of Clinical Microbiology 24, 47-51.

Clements, M. L., Subbarao, E. K., Fries, L. F., Karron, R. A., London, W. T. \& Murphy, B. R. (1992). Use of single-gene reassortant viruses to study the role of avian influenza A virus genes in attenuation of wild-type human influenza A virus for squirrel monkeys and adult human volunteers. Journal of Clinical Microbiology 30, 655-662.

Desselberger, U., Racaniello, V. R., Zazra, J. J. \& Palese, P. (1980). The $3^{\prime}$ and $5^{\prime}$-terminal sequences of influenza A, B and C virus RNA segments are highly conserved and show partial inverted complementarity. Gene 8, 315-328.

Digard, P., Block, V. C. \& Inglis, S. C. (1989). Complex formation between influenza virus polymerase proteins expressed in Xenopus oocytes. Virology 171, 162-169.

Gonzalez, S. \& Ortin, J. (1999 a). Distinct regions of influenza virus PB1 polymerase subunit recognize vRNA and cRNA templates. EMBO Journal 18, 3767-3775.

Gonzalez, S. \& Ortin, J. (1999 b). Characterization of influenza virus PB1 protein binding to viral RNA: two separate regions of the protein contribute to the interaction domain. Journal of Virology 73, 631-637.

Gonzalez, S., Zurcher, T. \& Ortin, J. (1996). Identification of two separate domains in the influenza virus PBI protein involved in the interaction with the PB2 and PA subunits: a model for the viral RNA polymerase structure. Nucleic Acids Research 24, 4456-4463.

Guan, Y., Shortridge, K. F., Krauss, S. \& Webster, R. G. (1999). Molecular characterization of $\mathrm{H} 9 \mathrm{~N} 2$ influenza viruses: were they the donors of the 'internal' genes of H5N1 viruses in Hong-Kong? Proceedings of the National Academy of Sciences, USA 96, 9363-9367.

Honda, A., Ueda, K., Nagata, K. \& Ishihama, A. (1988). RNA polymerase of influenza virus: role of NP in RNA chain elongation. Journal of Biochemistry 104, 1021-1026.

Kawaoka, Y., Krauss, S. \& Webster, R. G. (1989). Avian-to-human transmission of the PBI gene of influenza A viruses in the 1957 and 1968 pandemics. Journal of Virology 63, 4603-4608.

Li, M. L., Ramirez, B. C. \& Krug, R. M. (1998). RNA-dependent activation of primer RNA production by influenza virus polymerase: different regions of the same protein subunit constitute the two required RNA-binding sites. EMBO Journal 17, 5844-5852.

Lindstrom, S. E., Hiromoto, Y., Nerome, R., Omoe, K., Sugita, S., Yamasaki, Y., Takahashi, T. \& Nerome, K. (1998). Phylogenetic analysis of the entire genome of influenza A (H3N2) viruses from Japan: evidence for genetic reassortment of the six internal genes. Journal of Virology 72, 8021-8031.

Mandler, J., Muller, K. \& Scholtissek, C. (1991). Mutants and revertants of an avian influenza A virus with temperature-sensitive defects in the nucleoprotein and PB2. Virology 181, 512-519.

Murphy, B. R., Hinshaw, V. S., Sly, D. L., London, W. T., Hosier, N. T., Wood, F. T., Webster, R. G. \& Chanock, R. M. (1982). Virulence of avian influenza A viruses for squirrel monkeys. Infection and Immunity $\mathbf{3 7}$, 1119-1126.

Pleschka, S., Jaskunas, R., Engelhardt, O. G., Zurcher, T., Palese, P. \& Garcia-Sastre, A. (1996). A plasmid-based reverse genetics system for influenza A virus. Journal of Virology 70, 4188-4192.
Poch, O., Sauvaget, I., Delarue, M. \& Tordo, N. (1989). Identification of four conserved motifs among the RNA-dependent polymerase encoding elements. EMBO Journal 8, 3867-3874.

Sanz-Ezquerro, J. J., de la Luna, S., Ortin, J. \& Nieto, A. (1995). Individual expression of influenza virus PA protein induces degradation of coexpressed proteins. Journal of Virology 69, 2420-2426.

Scholtissek, C., Rohde, W., von Hoyningen, V. \& Rott, R. (1978). On the origin of the human influenza subtype $\mathrm{H} 2 \mathrm{~N} 2$ and $\mathrm{H} 3 \mathrm{~N} 2$. Virology 87 , 13-20.

Scholtissek, C., Hinshaw, V. S. \& Olsen, C. W. (1998). Influenza in pigs and their role as the intermediate host. In Textbook of Influenza, pp. 137-145. Edited by K. G. Nicholson, R. G. Webster \& A. J. Hay. Oxford: Blackwell Science.

Shi, L., Galarza, J. M. \& Summers, D. F. (1996). Recombinantbaculovirus-expressed PB2 subunit of the influenza A virus RNA polymerase binds cap groups as an isolated subunit. Virus Research 42, 1-9.

Snyder, M. H., Buckler-White, A. J., London, W. T., Tierney, E. L. \& Murphy, B. R. (1987). The avian influenza virus nucleoprotein gene and a specific constellation of avian and human virus polymerase genes each specify attenuation of avian-human influenza A/Pintail/79 reassortant viruses for monkeys. Journal of Virology 61, 2857-2863.

Suarez, D. L., Perdue, M. L., Cox, N., Rowe, T., Bender, C., Huang, J. \& Swayne, D. E. (1998). Comparisons of highly virulent H5N1 influenza A viruses isolated from humans and chickens from Hong Kong. Journal of Virology 72, 6678-6688.

Subbarao, E. K., London, W. \& Murphy, B. R. (1993). A single amino acid in the PB2 gene of influenza A virus is a determinant of host range. Journal of Virology 67, 1761-1764.

Subbarao, K., Klimov, A., Katz, J., Regnery, H., Lim, W., Hall, H., Perdue, M., Swayne, D., Bender, C., Huang, J., Hemphill, M., Rowe, T., Shaw, M., Xu, X., Fukuda, K. \& Cox, N. (1998). Characterization of an avian influenza A (H5N1) virus isolated from a child with a fatal respiratory illness [see comments]. Science 279, 393-396.

Tian, S. F., Buckler-White, A. J., London, W. T., Reck, L. J., Chanock, R. M. \& Murphy, B. R. (1985). Nucleoprotein and membrane protein genes are associated with restriction of replication of influenza A/Mallard/NY/78 virus and its reassortants in squirrel monkey respiratory tract. Journal of Virology 53, 771-775.

Tomic, M., Sunjevaric, I., Savtchenko, E. S. \& Blumenberg, M. (1990). A rapid and simple method for introducing specific mutations into any position of DNA leaving all other positions unaltered. Nucleic Acids Research 18, 1656.

Toyoda, T., Adyshev, D. M., Kobayashi, M., Iwata, A. \& Ishihama, A. (1996). Molecular assembly of the influenza virus RNA polymerase: determination of the subunit-subunit contact sites. Journal of General Virology 77, 2149-2157.

Webster, R. G. \& Bean, W. J., Jr (1998). Evolution and ecology of influenza viruses: interspecies transmission. In Textbook of Influenza, pp. 109-119. Edited by K. G. Nicholson, R. G. Webster \& A. J. Hay. Oxford: Blackwell Science.

Zürcher, T., de la Luna, S., Sanz-Ezquerro, J. J., Nieto, A. \& Ortín, J. (1996). Mutational analysis of the influenza virus $A / V i c t o r i a / 3 / 75$ PA protein: studies of interaction with $\mathrm{PBI}$ protein and identification of a dominant negative mutant. Journal of General Virology 77, 1745-1749.

Received 8 November 1999; Accepted 26 January 2000 